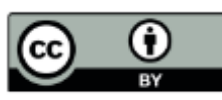

\title{
La colaboración público-privada en Salud en \\ España. El caso del Departamento de Torrevieja*
}

\section{Public-private collaboration in health in Spain. The case of the Department of Torrevieja}

\section{Colaboração público-privada em saúde em Espanha. $O$ caso do Departamento de Torrevieja}

Recibido: 30 de Mayo de 2019. Aceptado: 27 de Noviembre de 2019. Publicado: 1 de Octubre de 2020.

DOI: https://doi.org/10.11144/Javeriana.rgps19.cpps

\author{
David Vivas Orts ${ }^{a}$ \\ Universitat Politècnica de València, España \\ ORCID: https://orcid.org/0000-0003-0548-4756 \\ Carla Sancho Mestre \\ Universitat Politècnica de València, España \\ ORCID: https://orcid.org/0000-0001-9258-8937 \\ Mario Gómez Sacedón \\ Universitat Politècnica de València, España \\ ORCID: https://orcid.org/0000-0003-0182-9809 \\ Carlos Roche Vilanova \\ Universitat de València, España \\ ORCID: https://orcid.org/0000-0003-2356-2025
}

\begin{abstract}
Para citar este artículo Vivas D, Sancho C, Gómez M, Roche C. La colaboración público-privada en Salud en España. El caso del Departamento de Torrevieja. Rev Gerenc Polit Salud. 2020;19. https://doi.org/10.11144/Jav eriana.rgps 19.cpps
\end{abstract}

\footnotetext{
a Autor de correspondencia. Correo electrónico: davidvivs@gmail.com
} 


\section{Resumen}

El objetivo de este artículo es analizar la financiación y el movimiento de pacientes en el Departamento de Salud de Torrevieja en España. Situado en la Comunidad Valenciana, este Departamento se encuentra en una zona limítrofe con gran afluencia de turistas extranjeros, en la que el Gobierno regional constituyó un partenariado público-privado (PPP). Se utilizó información de las cuentas anuales de la concesionaria, de los presupuestos regionales y de los aplicativos sobre sanidad pública del territorio. Los resultados muestran, en primer lugar, un incremento constante en el gasto público, a pesar de no observarse un incremento total en la evolución de la población cubierta. Esto contrasta con el crecimiento menor del gasto público en los hospitales de gestión directa. En segundo lugar, el análisis de la población del territorio costero muestra una gran presencia de extranjeros y, en períodos estivales, una mayor carga asistencial causada por los desplazamientos de la población nacional y extrajera. Los resultados pretenden ser útiles para futuros PPP en hospitales de zonas limítrofes y turísticas.

Palabras clave: Financiación capitativa, concesión sanitaria, atención sanitaria población extranjera, comunidad limítrofe.

\section{Abstract}

This study aims to analyze the funding and movement of patients in the Torrevieja Health Department in Spain. Located in the Valencian Community, this Department is part of a border area with a large influx of foreign tourists, in which the regional government established a public-private partnership (PPP). Information from the concession company's annual accounts, regional budgets, and applications on public health in the territory was used. The results show, first, a constant increase in public spending, despite not observing a total increase in the evolution of the covered population. This observation contrasts with lower growth in public spending by hospitals with direct management. Second, the analysis of the population of the coastal territory shows a large presence of foreigners and, in summer periods, a greater care burden caused by the movements of the national and foreign populations. The results are intended to be useful for future PPPs in hospitals in border and tourist areas.

Keywords: Capitation funding, health concession, health care for foreign population, border community.

\section{Resumo}

O objetivo deste artigo é analisar o financiamento e circulação de pacientes no Departamento de Saúde de Torrevieja, na Espanha. Localizado na Comunidade Valenciana, este Departamento encontra-se em uma área limítrofe com grande afluxo de turistas estrangeiros, na qual o governo regional estabeleceu uma parceria público-privada (PPP). Foram utilizadas informações das contas anuais da concessionária, dos orçamentos regionais e dos aplicativos sobre saúde pública no território. Os resultados mostram, em primeiro lugar, um aumento constante na despesa pública, apesar de não se observar um aumento total na evolução da população coberta. Isto contrasta com o menor crescimento da despesa pública em hospitais gerenciados diretamente. Em Segundo lugar, a análise da população do território costeiro mostra uma grande presença de estrangeiros e, nos períodos de verão, um maior ônus assistencial causado pela circulação da população nacional e estrangeira. Os resultados pretendem ser úteis para futuras PPP em hospitais de áreas limítrofes e turísticas.

Palavras-chave: Financiamento por capitação, concessão dos cuidados da saúde, assistência médica à população estrangeira, comunidade limítrofe. 


\section{Introducción}

El presente artículo pretende realizar un análisis del partenariado público privado (PPP) desarrollado en el Departamento de Salud de Torrevieja dentro de la Comunidad Valenciana en España, una zona limítrofe con gran afluencia de extranjeros y de pacientes de otras áreas geográficas.

En España se ha establecido un modelo de repartición de las competencias y atribuciones de las distintas Administraciones Públicas en materia de Sanidad que se ha descentralizado progresivamente (1). Actualmente, se ha transferido completamente a todas las Comunidades Autónomas (entidades territoriales administrativas españolas) la gestión de la Sanidad (2). Con esta tendencia descentralizadora se ha desarrollado un marco legal que ha permitido la aparición de nuevos modelos de gestión de los servicios sanitarios, como especifica la Ley 15/1997, la cual permitió que se pudieran llevar a cabo concesiones administrativas en materia de sanidad. En la Comunidad Valenciana, en particular, se extendió el modelo de PPP comúnmente conocido como "modelo Alzira" en diferentes departamentos de Salud, ahora en proceso de reversión a la gestión pública (3).

El "modelo Alzira" implica que las entidades concesionarias -Uniones Temporales de Empresas (UTE) que generalmente reúnen aseguradoras, constructoras o bancos- se encargan tanto de la construcción y el mantenimiento de las infraestructuras como de la gestión de los servicios asistenciales (Centros de Salud y Centros de Especialidades y Hospitales), a cambio de una prima anual por cada persona cubierta pagada por el órgano competente de la Comunidad Autónoma de Valencia (Comunidad Valenciana). Esta prima anual se calcula de tal forma que el costo de la concesión sea menor que el costo de los servicios sanitarios con medios solamente públicos o menor que el costo de otras áreas geográficas que se puedan comparar (4).

El estudio se centra en un análisis del precio inicial del contrato y los movimientos de pacientes atendidos en el Departamento de Torrevieja bajo una PPP, y pretende ser útil en el establecimiento de futuros modelos de PPP en otras zonas limítrofes con gran afluencia de turistas extranjeros y nacionales. La población analizada serán los pacientes atendidos en el Departamento durante los años 2016, 2017 y 2018. Dicho Departamento tiene una población asignada por la que recibe una cápita anual. Sin embargo, también atiende pacientes no cápita de Departamento, por cuyas asistencias recibirá la correspondiente compensación económica, de acuerdo con las tarifas establecidas anualmente en la ley de tasas de acuerdo con los GRD (Grupos Relacionados de Diagnóstico). Se consideraron exclusivamente las asistencias que se refieren a ingresos hospitalarios, por considerarse una muestra representativa y por ser aquellas que generan un volumen económico más elevado.

La primera sección expondrá los materiales y métodos utilizados. La segunda sección se centrará en los fundamentos del modelo sanitario español y de la Comunidad Valenciana y del "modelo Alzira". La tercera sección analizará la financiación del departamento de Torrevieja como 
ejemplo de un departamento limítrofe con un número elevado de extranjeros y presentará los movimientos de los pacientes. Finalmente se expondrán las conclusiones.

\section{Materiales y métodos}

En primer lugar, el trabajo contextualizará el modelo sanitario español y concretamente el modelo Alzira en la Comunidad Valenciana, siguiendo un método narrativo y a partir de instrumentos normativos. Para ello, se utilizarán como fuentes principales la legislación estatal española y la autonómica de la región de la Comunidad Valenciana, así como las cuentas anuales de la adjudicataria. En segundo lugar, se realizará un análisis de datos respecto a la financiación de la concesión en Torrevieja y el flujo de pacientes y la población asignada en el territorio. Los datos serán extraídos tanto de las cuentas anuales de la concesionaria como de los presupuestos regionales del órgano competente y que financia la concesión, así como de las distintas bases de datos y aplicativos que regulan los diversos aspectos y características de la sanidad pública en la Comunidad Valenciana (bases de datos de la Conselleria de Sanitat de la Comunidad Valenciana y Aplicativo COMPAS).

\section{El modelo PPP en la Comunidad Valenciana}

\section{El modelo de gestión de la sanidad pública en la Comunidad Valenciana en España}

El modelo actual de la gestión de la sanidad pública en España se inició con la aprobación de la Ley 14/1986 General de Sanidad y la creación del Sistema Nacional de Salud; se financia con impuestos generales y es de cobertura universal (2). Desde entonces se han venido desarrollando progresivamente cambios que culminaron en 2003, con la Ley 16/2003 de Cohesión de la Calidad del Sistema Nacional de Salud, con el proceso de transferencias de las competencias en materia de sanidad a las Comunidades Autónomas (CCAA), cada una de las cuales cuenta con un Servicio de Salud como estructura básica administrativa (5). Este proceso de descentralización se llevó a cabo para poder acercar la gestión sanitaria a la población y conseguir un mejor funcionamiento de las Administraciones Públicas $(6,7)$. El reparto actual de competencias es el siguiente (8):

Competencias del Estado: bases y coordinación general de la sanidad (condiciones y requisitos mínimos de los servicios sanitarios públicos), acuerdos sanitarios internacionales (vigilancia y control de riesgos derivados de tránsito de mercancías y tráfico internacional de viajeros) y legislación, evaluación y control de productos farmacéuticos.

Competencias de las CCAA: planificar la salud pública, y gestionar los Servicios de Salud.

Competencias de los municipios: salubridad y colaboración con el resto de administraciones públicas. 
El proceso de transferencia total de atribuciones a las CCAA entre 1986 y 2003 estuvo acompañado de un incremento constante del gasto sanitario público y de un necesario control de éste. Entre 1991 y 2010, el gasto sanitario público creció a una tasa media anual de 7,5\%, pasando de 18.000 millones de euros a 70.000 millones de euros (9). Los estudios sobre el control del gasto exponen dos alternativas de realización: (i) el modelo alemán, recortando directamente el gasto sanitario público, o (ii) el modelo de Gran Bretaña o Suecia, reformando la gestión sanitaria hacia modelos más eficientes (4). La elección del segundo modelo derivó en la Ley 15/1997 sobre Habilitación de Nuevas Formas de Gestión del Sistema Nacional de Salud, la cual otorgó la competencia a las Administraciones Públicas de desarrollar nuevas formas de colaboración y gestión sanitarias, mediante acuerdos, convenios o concesiones administrativas.

El Sistema Valenciano de Salud integra todos los centros, servicios y establecimientos de la Comunidad Valenciana destinados a hacer efectivo el derecho a la salud. Se organiza y ordena en Departamentos de Salud (Ley 10/2014, de Salud de la Comunidad Valenciana) (10). Estos Departamentos son las demarcaciones geográficas en las que queda dividido el territorio de la Comunidad Valenciana para efectos sanitarios, pudiendo variar el contingente de su población cubierta con el objetivo de prestar una asistencia sanitaria y sociosanitaria eficaz. Cada provincia, en todo caso, tendrá, como mínimo, un Departamento de Salud, que se subdivide en Zonas Básicas de Salud. En la actualidad existe un total de 24 Departamentos de Salud subdivididos en diferentes Zonas Básicas de Salud (4).

Los servicios sanitarios en cada Departamento de Salud de cada región de España se organizan en dos niveles. El primer nivel, Atención Primaria, tiene gran accesibilidad y aborda los problemas de salud frecuentes. Los dispositivos de asistencias de la Atención Primaria son los Centros de Salud. El segundo nivel, la Atención Especializada, se presta en Centros de Especialidades y Hospitales, de manera ambulatoria o en régimen de ingreso. Cada Departamento de Salud dispone de un Hospital general como referente de la Atención Especializada, y cada Zona Básica de Salud dispone de un Centro de Salud.

Para el desarrollo de la estructura del sistema sanitario valenciano, en virtud del marco estatal, se establece que la gestión sanitaria de los centros se lleva a cabo preferentemente bajo la gestión directa, sin perjuicio de que la Comunidad Valenciana pueda establecer concesiones administrativas para la gestión de la sanidad cuando así lo considere, siguiendo la Ley 15/1997.

\section{El modelo de PPP de Alzira y sus fundamentos}

En este contexto de descentralización y fomento de la gestión indirecta en la sanidad pública española, el Partido Popular (PP) de la Comunidad Valenciana, situado a la derecha política, apoyándose en políticas de racionalidad gerencial de New Public Management, implantó el "modelo Alzira" en varios Departamentos de Salud $(11,12)$. Esta sección expone un análisis histórico de ello. 
Tras la entrada en vigor de la Ley 15/1997, el PP propuso que en el único Departamento de Salud de la Comunidad Valenciana que no poseía un Hospital para Atención Especializada (comarca de Alzira) se firmara un PPP. Ante estos casos, las formas de PPP que se han aplicado más recientemente son de dos tipos (13). Por un lado, el sector público puede firmar con una entidad privada, a cambio de un canon anual, contratos de obra pública (Iniciativa de Financiación Privada: PFI, por sus siglas en inglés). En ellos el adjudicatario se obliga a diseñar y construir una infraestructura sanitaria, así como en ciertos casos también a gestionar los servicios no clínicos (seguridad, lavandería, limpieza, etc.).

Por ejemplo, en Reino Unido, un país líder en PPP, se desarrolló previamente la Iniciativa de Financiación Privada (PFI, por sus siglas en inglés), siendo éste el uso más común de este tipo de modelos. Este toma un enfoque de infraestructura, donde el concesionario construye y mantiene el hospital, incluida una transferencia de riesgos en el mantenimiento de las instalaciones del gobierno al concesionario (14). El modelo PFI se ha aplicado a situaciones en las que el sector público tiene que emprender grandes proyectos de infraestructura con crecientes restricciones presupuestarias (15). Sin embargo, puede resultar en que los gobiernos tengan menos flexibilidad presupuestaria en los tiempos de consolidación fiscal como resultado de transferir el riesgo y trasladar el costo de capital de la construcción a través de pagos fijos a largo plazo al socio privado (16).

Y por otro lado, el sector público puede firmar concesiones administrativas con otras entidades, las cuales además de encargarse de la construcción y diseño de las infraestructuras el sector público se encargan de la gestión de los servicios asistenciales. A cambio de la obra y prestación de servicios, el sector público establece una prima por cada persona cubierta y año, fijada para que no sobrepase el costo público.

Cabe destacar las bases conceptuales del modelo y los principales aspectos de los pliegos del contrato para comprender el "modelo Alzira" (17).

A continuación se resumen las características fundamentales del modelo aplicables en cada una de las cinco concesiones, siguiendo a varios autores $(14,15)$ :

Financiación pública (el Gobierno regional valenciana abona, a principios de año, a la concesionaria un pago capitativo, que toma como referencia el crecimiento anual del presupuesto sanitario expresado en los presupuestos regionales y que en ningún caso podrá ser inferior a la actualización en función del Índice de Precios al Consumo (IPC), ni superior al porcentaje de incremento anual del gasto sanitario público consolidado del estado español para dicho ejercicio. Concepto denominado cápita provisional o cápita presupuestaria. La cuantía resultante, multiplicada por la población protegida asignada a la empresa concesionaria conforme a los criterios en materia de aseguramiento sanitario, será el precio inicial del contrato del Departamento. 
Las partidas presupuestarias que se tienen en cuenta para establecer el pago capitativo son las relativas a los gastos de personal, la compra de bienes y gastos en funcionamiento y de inversiones de los programas de asistencia Sanitaria, Salud Mental y Atención Sanitaria de Media y Larga Estancia e Información para la Salud. Existe además una partida sobre Prestaciones Externas que sólo tiene en cuenta el presupuesto asignado a la compra de bienes y gastos de funcionamiento, excluyendo de ésta las prestaciones de oxigenoterapia y transporte, por quedar fuera del ámbito contractual.

La cápita presupuestaria que se establece a principios del año se revisa en el momento de la liquidación oportuna de ese ejercicio. En esta revisión capitativa, se tendrá en cuenta, además de los límites que comentamos anteriormente (IPC y Gasto Sanitario Público Consolidado del Estado) el Gasto Real Sanitario Capitativo de la Comunidad Valenciana.

Revisado el precio, se debe revisar la población protegida tenida en cuenta para establecer el precio inicial del contrato del ejercicio, ya que ésta fue la tomada como referencia el 30 de septiembre del ejercicio anterior. Para ello se deberá tomar como referencia, la población protegida a fecha 31 de diciembre del ejercicio anterior.

Facturación inter-centros: en el caso de que un paciente del Departamento de Gestión Privada acuda a un centro sanitario de otro Departamento de Gestión Directa, la concesionaria paga el $100 \%$ del costo del paciente. Si, por el contrario, la empresa concesionaria recibe a un paciente de un Departamento de Gestión Pública, la Administración paga a la concesionaria según lo estipulado en el contrato, conforme a la Ley de Tasas y Precios Públicos vigente. Se aplica un Coeficiente de Transferencia de Servicios que actúa como un corrector del precio a aplicar por el concesionario en su facturación y se establecerá en función de la oferta del adjudicatario, el cual es de $85 \%$ en Torrevieja.

Control público: la Administración Sanitaria nombra a una persona (funcionario/estatutario), para que ejerza como representante de esta en el Hospital y centros dependientes, para ejercer, entre otras, las siguientes funciones:

Controlar, aceptar y denegar las propuestas de ingreso programado de los enfermos no pertenecientes a la población de referencia.

Ordenar el traslado de pacientes en casos de ingresos de otras demarcaciones que considere deben ser remitidos al hospital de referencia de su zona de residencia.

Supervisar la realización de las encuestas o sondeos de opinión.

Recibir las sugerencias, quejas y reclamaciones. 
Ejercer la Jefatura orgánica del Personal dependiente de la Administración Sanitaria en los centros gestionados por el concesionario.

Verificar y en su caso devolver la facturación del concesionario de actos médicos realizados a pacientes no correspondientes a la población protegida por el contrato.

La Administración, vigilará a través del Comisionado, de sus propios servicios técnicos, y de las auditorias que ordene la correcta ejecución del Plan de Inversiones vigente.

Con el propósito de asegurar una explotación equilibrada de la concesión y el máximo control de la Administración, se limita la Tasa Interna de Rentabilidad (TIR) del proyecto al 7,5\% anual sobre la inversión realizada a lo largo de la duración de la concesión.

Sin perjuicio de las competencias de los órganos de fiscalización interna y externa del Gobierno regional, un auditor externo -nombrado por consenso entre Administración y concesionariorevisará cada cinco años la rentabilidad conseguida por el concesionario en ese periodo, y las variaciones de rentabilidad (TIR) que hubiere se ajustarán mediante la programación de inversiones.

Propiedad pública: finalizado el plazo de la concesión el servicio revertirá a la Administración, así como la propiedad libre de cargas del hospital y todo su equipamiento. El adjudicatario entregará el edificio, instalaciones y equipamiento a que este contrato le obliga, en el estado de conservación y funcionamiento adecuados.

Prestación privada: la prestación sanitaria la gestiona la adjudicataria. Existe una transferencia teórica de riesgo de la Administración a la adjudicataria.

En 2003, el Gobierno regional valenciano adjudicó por concurso a "Torrevieja Salud UTE Ley 18/82" un contrato de 15 años, para el diseño y la construcción del hospital, así como para la gestión de Servicios Públicos en la modalidad de concesión (16). La concesión abrió sus puertas el día 16 de octubre de 2006, y la fecha de finalización prevista es el 16 de octubre de 2021.

En cuanto a si el contrato va a ser o no prorrogado durante 5 años más (posibilidad que se contempla en el propio contrato), existen dudas a causa de la incertidumbre política actual y el vínculo del modelo con la misma. De hecho, ya se ha dado el primer caso de finalización del contrato sin ejecución de la prórroga. En 2015, hubo un cambio de Gobierno central en la Comunidad Valenciana, que desarrolló un nuevo plan estratégico para la Salud en el territorio (17). El Partido Socialista del País Valenciano (PSPV), de ideología de izquierdas, llevaba en su programa electoral la no renovación de los contratos de las concesiones para así volver a una gestión pública de la sanidad en el territorio valenciano. En 2018, el contrato de la concesión 
de Alzira expiró y comenzó a operar la reversión, que vino acompañada de conflictos legales y económicos entre el Gobierno regional, la adjudicataria y los trabajadores (18).

La empresa concesionaria objeto de estudio, a la vista de sus resultados económicos, estaría interesada en que esta prórroga tuviese lugar. Concretamente, la empresa concesionaria ha logrado unos beneficios acumulados durante el periodo 2006 a 2017 (último del que se dispone de cuentas anuales auditadas) de más de 62 millones de euros, que se distribuyen conforme muestra la figura 1.

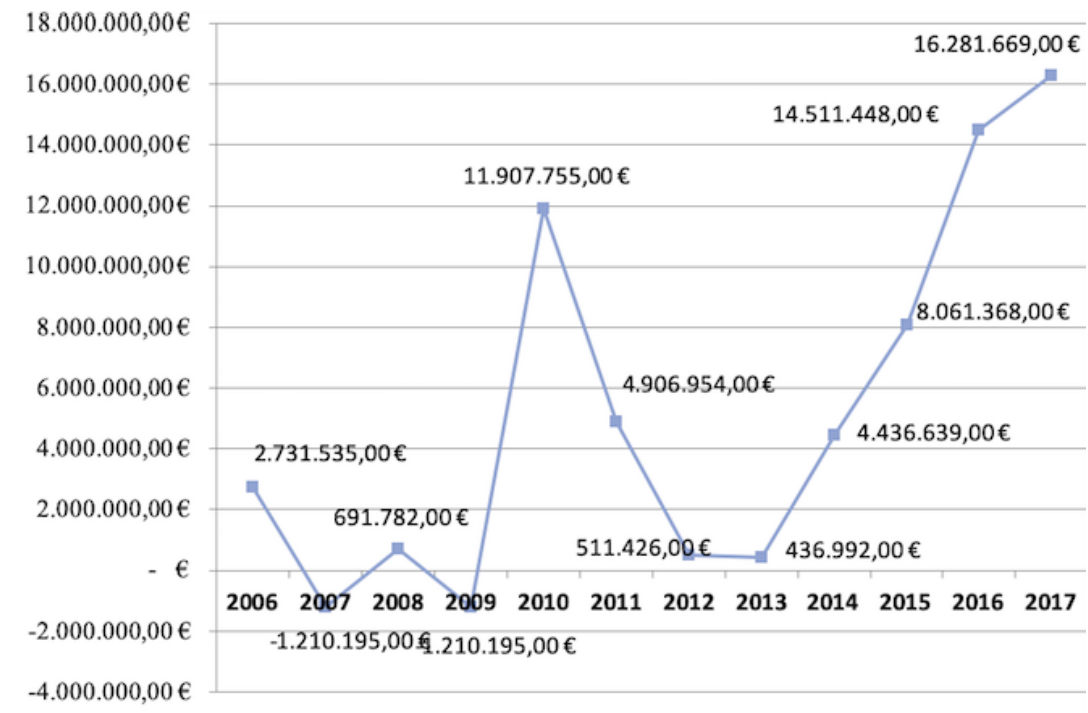

Figura 1 Evolución beneficios Torrevieja Salud UTE Ley 18/82, 2006-2017

Fuente: elaboración propia con base en las cuentas anuales auditadas de Torrevieja Salud UTE Ley 18/82.

\section{Resultados}

\section{Análisis de la financiación del Dpto. de Torrevieja según asignación cápita y su evolución}

La financiación del Departamento depende de dos variables (la población y el ingreso capitativo anual) (15). Con ello, se obtiene el montante anual para que la empresa preste el servicio, que se dividirá en pagos mensuales como pagos a cuenta. Posteriormente, el precio final del contrato se ajustará mediante una liquidación anual entre la concesionaria y la Administración (19). En este subapartado se analiza tanto la evolución de la cápita como de la población, para observar la tendencia del ingreso capitativo anual total.

Como se ha explicado en apartados anteriores, la forma de financiación se basa en el pago de una cápita anual por paciente. En la figura 2 se muestra la evolución del pago anual por paciente que crece unos $300 €$ a lo largo del periodo analizado, lo cual representa más del $60 \%$ respecto al importe del año 2006. 


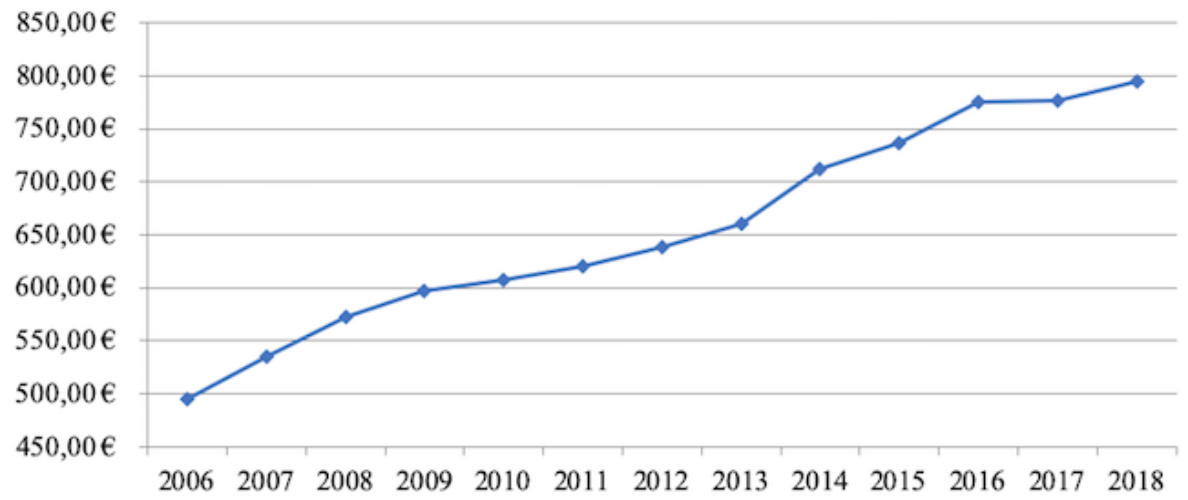

Figura 2 Evolución cápita, 2006-2018

Fuente: Conselleria de Sanidad.

La Sindicatura de Cuentas de la Generalidad Valenciana expresado la idea de que el pago capitativo resulta en un ahorro del gasto público medio en comparación con hospitales de gestión pública en torno al 30\% (20). Sin embargo, el crecimiento anual de la prima per cápita entre 2006 y 2018 resulta mayor que el crecimiento anual del gasto medio por paciente en un hospital de gestión pública en la misma Comunidad Valenciana. Concretamente, el crecimiento acumulado ha sido de un $74 \%$ en los hospitales bajo un PPP, mientras que en el resto ha sido de un $40 \%$ (ver la figura 3).

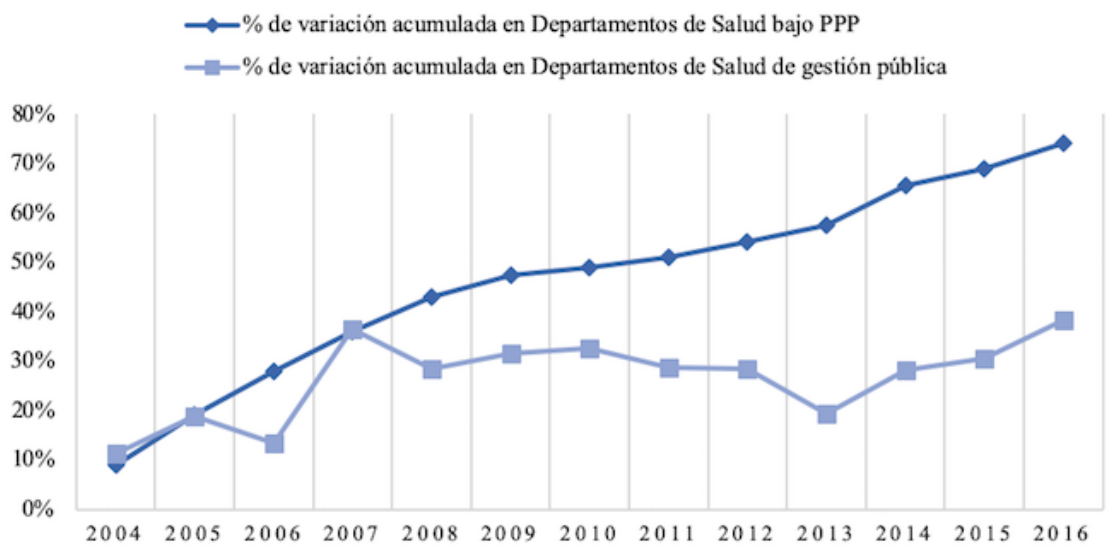

Figura 3 Evolución del crecimiento acumulado en un PPP y no PPP, 2004-2016 Fuente: elaboración propia con base en los presupuestos generales del Estado y la Conselleria de Sanidad.

Por otra parte, y como se puede apreciar en la figura 4, la evolución de la población protegida en el departamento de Torrevieja ha experimentado variaciones. En 2018 la cifra de población protegida es superior a la del inicio de la concesión en 2006, pero solamente en un 4\% (156.067 en 2018 por 150.067 en 2006). 


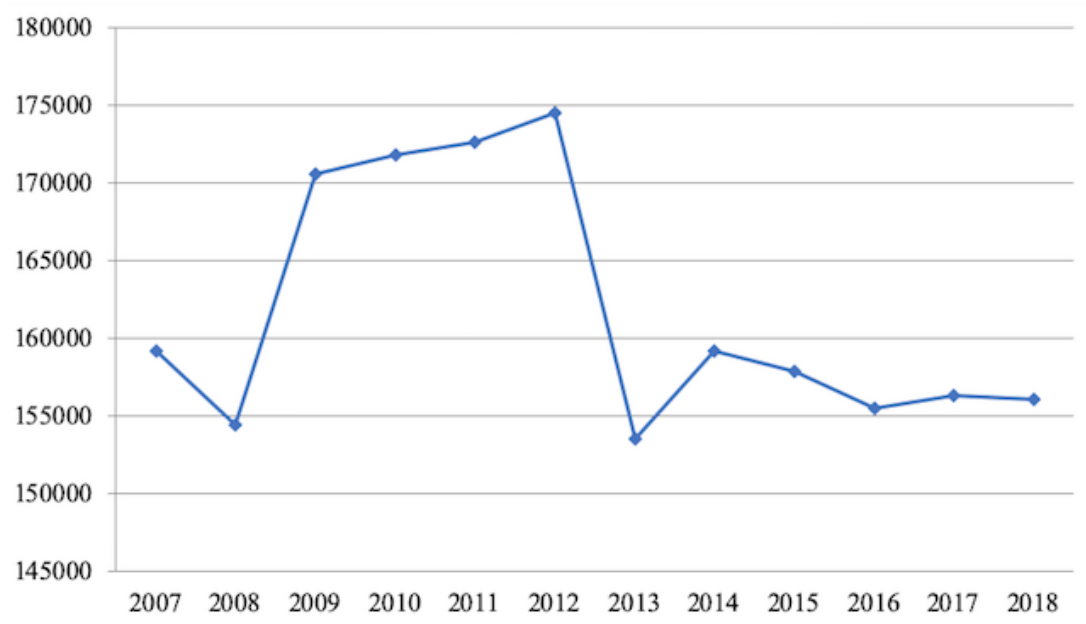

Figura 4 Evolución población 2006-2018

Fuente: Sistema de información poblacional, Conselleria de Sanidad.

Llama la atención el decremento de población a partir de 2012, si bien esta misma tendencia se aprecia en la totalidad del resto de Departamentos de Salud y Comunidades Autónomas. Por tanto, la hipotética situación de descenso de la "población protegida" de la concesión de Torrevieja se podría atribuir a una coyuntura general de descenso de la población residente en la Comunidad Valenciana (tanto española como extranjera) y en el resto de comunidades autónomas, máxime en un escenario de crisis económica profunda.

En la figura 5 se muestra el importe total (producto de la población y la cápita por persona), que por tanto evoluciona en función de ambas variables. El precio inicial del contrato ha alcanzado su importe máximo en el año 2018, siendo el ejercicio 2013 el que obtuvo un importe más bajo debido al decremento de población mencionado anteriormente. En esencia, la tendencia es de crecimiento constante anual, con la excepción de 2013. 


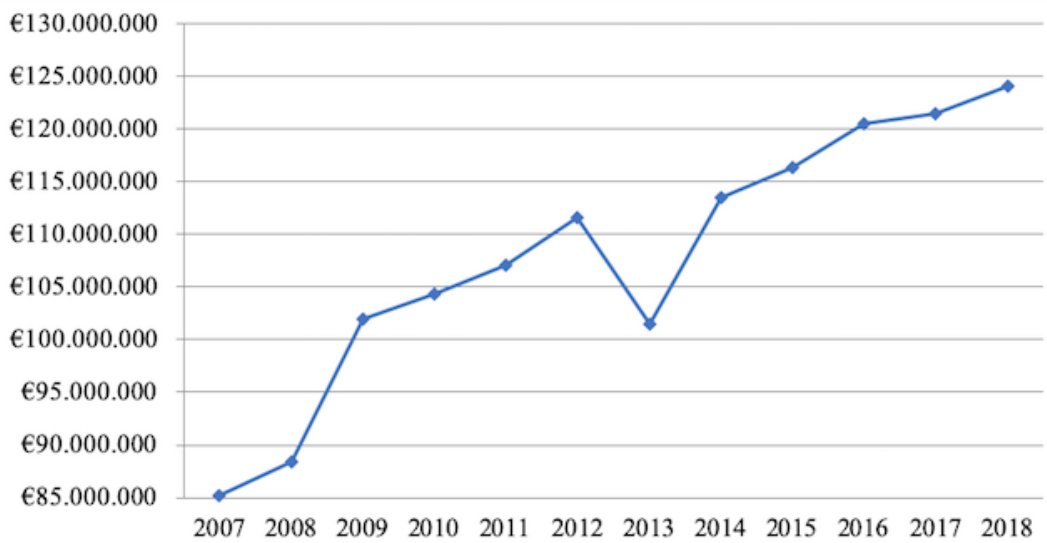

Figura 5 Evolución ingreso capitativo, 2007-2018

Fuente: Sistema de información poblacional, Conselleria de Sanidad.

\section{Análisis de los pacientes no cápita atendidos por la concesión}

Antes de valorar el movimiento de pacientes hacia la concesión de Torrevieja, es interesante mostrar la población residente en este municipio. Según los datos del padrón del año 2018 obtenidos de la página web de la Diputación de Alicante (21), la población total residente y empadrona en Torrevieja se sitúa en 82.599 personas. Siendo la población nacida fuera de la Comunidad Valenciana la mayoría de residentes en dicho municipio 22.915 nacidos en distinta Comunidad y 36.250 en el extranjero, frente a los 23.434 nacidos en la Comunidad Valenciana (CV), lo que resalta su peculiar situación demográfica.

En este subapartado se analiza el movimiento de pacientes hacia la concesión de Torrevieja para visualizar su especial situación geográfica con respecto a otros Departamentos de Salud de la Comunidad Valenciana (CV). Se realizará un análisis en función de la procedencia de los pacientes, desplazados nacionales o extranjeros, haciendo especial hincapié en la estacionalidad y distinguiendo entre números de asistencias e importes. Se han seleccionado para el análisis las hospitalizaciones para los años 2016, 2017 y 2018. Es decir, los pacientes que han tenido algún ingreso hospitalario a lo largo de los tres años. Para ello se ha obtenido la información del Aplicativo COMPAS de la Conselleria de Sanidad, aplicativo que se utiliza para la compensación de asistencias entre Departamentos de Salud.

El motivo de seleccionar este Departamento de Salud no es otro que su situación geográfica, limítrofe con la Comunidad Autónoma de Murcia y sobretodo, ciudad costera caracterizada por la gran afluencia de turistas en gran parte de otros países de la Unión Europea. 
Se han analizado los pacientes que no pertenecen a la población protegida de la concesionaria (pacientes no cápita por los que no se cobra cápita anual), en función de su origen y en el ámbito del ingreso hospitalario durante el periodo analizado. Los resultados de dicho análisis se presentan en la tabla 1 y la figura 6 .

Tabla 1 Datos origen pacientes no cápita ingresados en Torrevieja 2016-201

\begin{tabular}{|l|c|c|c|c|c|c|}
\hline & \multicolumn{2}{|c|}{2016} & \multicolumn{2}{c|}{2017} & \multicolumn{2}{c|}{2018} \\
\hline \multicolumn{1}{|c|}{ Origen } & $\begin{array}{c}\text { Pacientes } \\
\text { ingresados }\end{array}$ & $\%$ & $\begin{array}{c}\text { Pacientes } \\
\text { ingresados }\end{array}$ & $\%$ & $\begin{array}{c}\text { Pacientes } \\
\text { ingresados }\end{array}$ & $\%$ \\
\hline Dpto. Público & 332 & 14,15 & 310 & 14,08 & 306 & 12,40 \\
\hline Extranjeros & 1.190 & 50,72 & 1.173 & 53,27 & 1.291 & 52,33 \\
\hline Otras CCAA & 792 & 33,76 & 658 & 29,88 & 816 & 33,08 \\
\hline Otros & 32 & 1,36 & 61 & 2,77 & 54 & 2,19 \\
\hline Total general & 2.346 & 100 & 2.202 & 100 & 2.467 & 100 \\
\hline
\end{tabular}

Otras CCAA: Modalidad aseguramiento FSS11 a FSS144, No Empadronados.

Extranjeros: Modalidad aseguramiento FEX1 a FEX5, No Empadronados.

Dpto. Público: Empadronados en CV y asignado a un Dpto. Salud de Gestión Directa.

Otros: No Empadronados con modalidades distintas de Otras CCAA y Extranjeros.

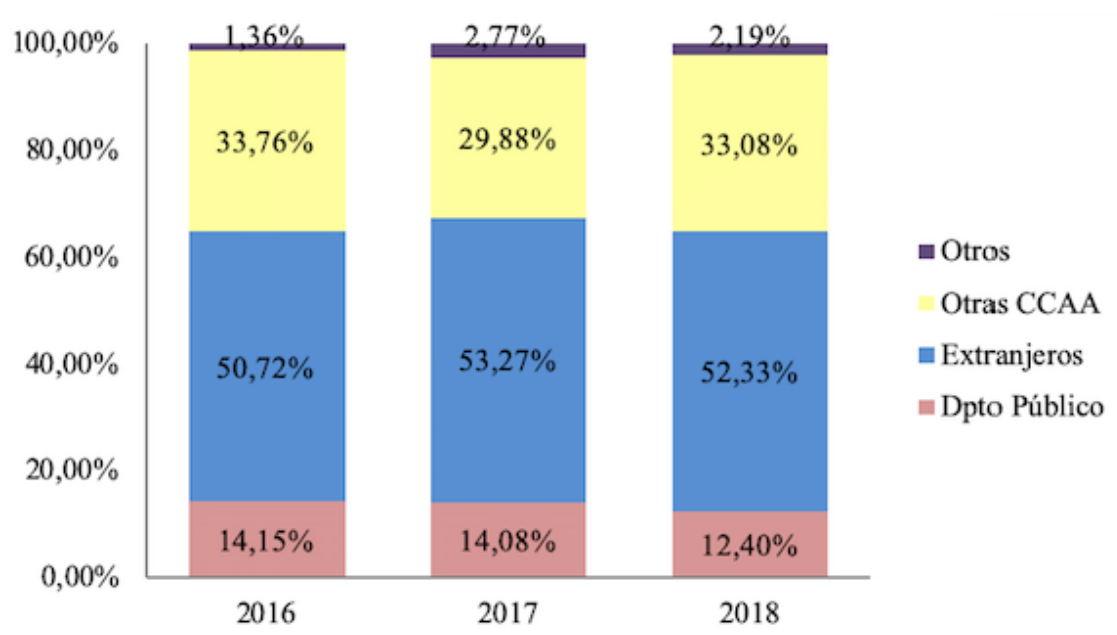

Figura 6 Origen pacientes no cápita ingresados en Torrevieja, 2016-2018 Fuente: aplicativo COMPAS Conselleria de Sanidad.

Como queda reflejado en la figura 6 , el $80-85 \%$ de los pacientes que son ingresados en el Departamento de Salud de Torrevieja para el periodo analizado, corresponden a pacientes extranjeros ( $52 \%$ de media para los tres años analizados) y Otras CCAA ( $32 \%$ de media para el 
periodo 2016-2018), lo que demuestra la peculiar situación geográfica de esta concesión, alejada del resto de Departamentos Públicos de la Comunidad Valenciana y con la mayoría de pacientes no cápita, no empadronados en la propia $\mathrm{CV}$.

En cuanto al volumen económico generado por estas asistencias en términos porcentuales, alrededor del $55 \%$ corresponden a pacientes extranjeros y un $30 \%$ a pacientes de otras comunidades (tabla 2). Solamente el $12 \%$ de la facturación se debe a la atención prestada a pacientes de los departamentos de la Comunidad Valenciana gestionados bajo el régimen de gestión directa, que en el periodo comprendido entre 2016 y 2018 eran 19. Esta es una cifra realmente baja que se explica por la ya comentada situación geográfica particular de este Departamento de Salud.

Tabla 2 Importe Ingresos Hospitalarios Torrevieja, 2016-2018

\begin{tabular}{|l|c|c|c|}
\hline & \multicolumn{3}{|c|}{ Importe Ingresos Hospitalarios (\%) } \\
\hline & $\mathbf{2 0 1 6}$ & $\mathbf{2 0 1 7}$ & $\mathbf{2 0 1 8}$ \\
\hline Dpto. Público & 12,32 & 12,29 & 11,11 \\
\hline Extranjeros & 56,21 & 56,99 & 53,79 \\
\hline Otras CCAA & 30,04 & 27,66 & 31,94 \\
\hline Otros & 1,43 & 3,06 & 3,16 \\
\hline Total general & 100,00 & 100,00 & 100,00 \\
\hline
\end{tabular}

Fuente: aplicativo COMPAS Conselleria de Sanidad

Dado que esta singularidad está relacionada directamente con el turismo, se han analizado estos movimientos incluyendo el factor estacional. Si nos encontrásemos en un caso normal, cabría esperar que el número de asistencias se distribuyese equitativamente a lo largo del año, es decir, aproximadamente un $25 \%$ cada trimestre. Sin embargo, se aprecia claramente como en el primer y último trimestre del año, los ingresos son inferiores al $25 \%$ mientras que para el segundo y el tercero se observan incrementos en cuanto al número de asistencias que alcanzan su máximo en el tercer trimestre (más del $35 \%$ de las asistencias anuales), coincidente con la época estival que suele traer aparejada mayor afluencia turística. 
Tabla 3 Asistencias con ingreso hospitalarios pacientes no Cápita Torrevieja 2016-2018 (trimestre)

\begin{tabular}{|l|c|c|c|c|}
\hline & \multicolumn{4}{|c|}{ Asistencias por trimestres periodo 2016-2018 } \\
\hline \multicolumn{1}{|c|}{ Origen } & 1 & 2 & 3 & 4 \\
\hline Dpto. Público & 217 & 245 & 353 & 210 \\
\hline Extranjeros & 985 & 1.034 & 1.017 & 884 \\
\hline Otras CCAA & 322 & 458 & 1.327 & 287 \\
\hline Otros & 38 & 26 & 66 & 33 \\
\hline Total general & 1.562 & 1.763 & 2.763 & 1.414 \\
\hline
\end{tabular}

Fuente: aplicativo COMPAS Conselleria de Sanidad.

Del mismo modo, si analizamos los datos económicos por trimestre y origen de los pacientes detectamos un efecto en el porcentaje de los pacientes extranjeros (tabla 4). Éste es más alto en los trimestres 1 y 4 en los que representan la mayoría de los recursos económicos. Sin embargo, en el en el tercer trimestre del año, el porcentaje de los extranjeros suponen un peso muy inferior, que no alcanza el $40 \%$ en el periodo julio-septiembre. Esto se explica porque este perfil de pacientes vive durante todo el año en la zona y por tanto, se le atiende de forma regular durante todo el año natural tanto en número de asistencias como en importe de ingreso.

Sin embargo, en el segundo y tercer trimestre, al crecer el volumen total de asistencias por la llegada de turistas de otras comunidades (el número de asistencias se llega a triplicar respecto a otros trimestres), los importes de los extranjeros que se mantienen constantes en términos absolutos, y pasan a tener un peso más bajo en términos relativos (tabla 4, figura 7).

Tabla 4 Datos importe trimestral ingresos hospitalarios pacientes no Cápita Torrevieja 2016-2018 (en porcentajes)

\begin{tabular}{|l|c|c|c|c|}
\hline & \multicolumn{5}{|c|}{ Importes ingres hospitalarios por trimestres, 2016-2018 (\%) } \\
\hline \multicolumn{1}{|c|}{ Origen } & 1 & 2 & 3 & 4 \\
\hline Dpto. Público & 12,96 & 11,30 & 11,30 & 12,74 \\
\hline Extranjeros & 66,07 & 62,28 & 39,75 & 64,39 \\
\hline Otras CCAA & 17,09 & 24,80 & 46,61 & 20,38 \\
\hline Otros & 3,88 & 1,62 & 2,34 & 2,49 \\
\hline Total general & 100,00 & 100,00 & 100,00 & 100,00 \\
\hline
\end{tabular}

Fuente: aplicativo COMPAS Conselleria de Sanidad. 


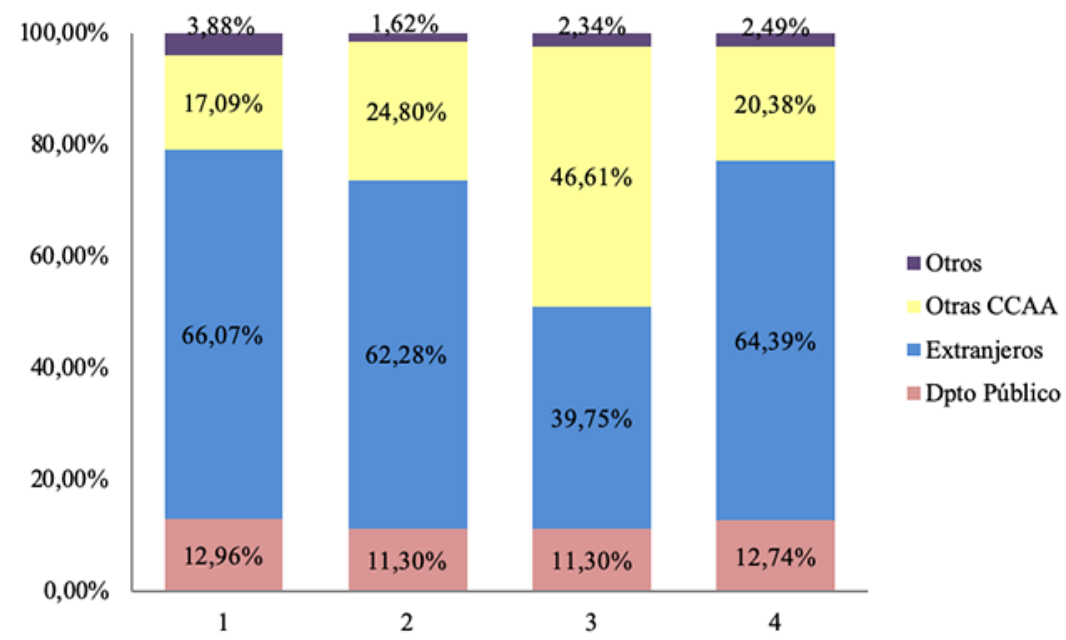

Figura 7 Importe trimestral ingresos hospitalarios pacientes no Cápita Torrevieja 2016-2018 (en porcentajes) Fuente: aplicativo COMPAS Conselleria de Sanidad.

En la tabla 5 se ha elaborado una clasificación en función del país de procedencia del paciente Los datos son obtenidos del GESDATA de la Diputación de Alicante (21). La provincia con mayor población ${ }^{1}$ británica de España es Alicante. Provincia en la que se encuentra la localidad de Torrevieja. Como no podría ser de otro modo, Reino Unido ocupa el primer puesto con una media del 43,68\% seguido a mucha distancia de Suecia $(11,81 \%)$, Alemania $(10,19 \%)$ y Noruega (7,73\%). Prácticamente la totalidad de los pacientes son de origen europeo, salvo casos aislados de Rusia, Nueva Zelanda y Chile. Es evidente, como ya hemos comentado anteriormente que el turismo juega un papel fundamental en esta situación, ciudadanos europeos de países próximos que vienen a España en busca de buen tiempo y servicios turísticos de primer nivel. 
Tabla 5 Pacientes extranjeros clasificados por país de origen

\begin{tabular}{|c|c|c|c|c|c|c|c|}
\hline & \multicolumn{2}{|c|}{2016} & \multicolumn{2}{|c|}{2017} & \multicolumn{2}{|c|}{2018} & \multirow{2}{*}{$\begin{array}{c}\text { Media } \\
\text { Periodo } \\
2016-2018\end{array}$} \\
\hline Extranjeros & $\begin{array}{l}\text { Pacientes } \\
\text { ingresados }\end{array}$ & $\%$ & $\begin{array}{l}\text { Pacientes } \\
\text { ingresados }\end{array}$ & $\%$ & $\begin{array}{l}\text { Pacientes } \\
\text { ingresados }\end{array}$ & $\%$ & \\
\hline 125: Reino Unido & 538 & 45,21 & 508 & 43,31 & 549 & 42,53 & 43,68 \\
\hline 131: Suecia & 151 & 12,69 & 135 & 11,51 & 145 & 11,23 & 11,81 \\
\hline 126: Alemania & 128 & 10,76 & 118 & 10,06 & 126 & 9,76 & 10,19 \\
\hline 120: Noruega & 98 & 8,24 & 100 & 8,53 & 83 & 6,43 & 7,73 \\
\hline 113: Irlanda & 63 & 5,29 & 66 & 5,63 & 67 & 5,19 & 5,37 \\
\hline 103: Bélgica & 47 & 3,95 & 73 & 6,22 & 82 & 6,35 & 5,51 \\
\hline 109: Finlandia & 35 & 2,94 & 46 & 3,92 & 52 & 4,03 & 3,63 \\
\hline 110: Francia & 34 & 2,86 & 37 & 3,15 & 36 & 2,79 & 2,93 \\
\hline 121: Países Bajos & 25 & 2,10 & 17 & 1,45 & 20 & 1,55 & 1,70 \\
\hline 114: Islandia & 22 & 1,85 & 20 & 1,71 & 47 & 3,64 & 2,40 \\
\hline 107: Dinamarca & 15 & 1,26 & 15 & 1,28 & 18 & 1,39 & 1,31 \\
\hline 132: Suiza & 8 & 0,67 & 10 & 0,85 & 11 & 0,85 & 0,79 \\
\hline 102: Austria & 7 & 0,59 & 4 & 0,34 & 6 & 0,46 & 0,46 \\
\hline 122: Polonia & 6 & 0,50 & 7 & 0,60 & 12 & 0,93 & 0,68 \\
\hline 117: Luxemburgo & 3 & 0,25 & 2 & 0,17 & 0 & 0,00 & 0,14 \\
\hline 104: Bulgaria & 2 & 0,17 & 1 & 0,09 & 6 & 0,46 & 0,24 \\
\hline 112: Hungria & 2 & 0,17 & 1 & 0,09 & 1 & 0,08 & 0,11 \\
\hline 115: Italia & 2 & 0,17 & 3 & 0,26 & 15 & 1,16 & 0,53 \\
\hline 142: Lituania & 1 & 0,08 & 3 & 0,26 & 3 & 0,23 & 0,19 \\
\hline 143: República Checa & 1 & 0,08 & 3 & 0,26 & 1 & 0,08 & 0,14 \\
\hline 144: Eslovaquia & 1 & 0,08 & 2 & 0,17 & 0 & 0,00 & 0,08 \\
\hline 504: Nueva Zelanda & 1 & 0,08 & 0 & 0,00 & 0 & 0,00 & 0,03 \\
\hline 123: Portugal & 0 & 0,00 & 0 & 0,00 & 1 & 0,08 & 0,03 \\
\hline 128: Rumania & 0 & 0,00 & 1 & 0,09 & 4 & 0,31 & 0,13 \\
\hline 136: Letonia & 0 & 0,00 & 0 & 0,00 & 1 & 0,08 & 0,03 \\
\hline 141: Estonia & 0 & 0,00 & 0 & 0,00 & 3 & 0,23 & 0,08 \\
\hline 146: Croacia & 0 & 0,00 & 0 & 0,00 & 1 & 0,08 & 0,03 \\
\hline 154: Rusia & 0 & 0,00 & 1 & 0,09 & 0 & 0,00 & 0,03 \\
\hline 344: Chile & 0 & 0,00 & 0 & 0,00 & 1 & 0,08 & 0,03 \\
\hline Total & 1.190 & 100,00 & 1.173 & 100,00 & 1.291 & 100,00 & 100,00 \\
\hline
\end{tabular}

Fuente: Aplicativo COMPAS Conselleria de Sanidad.

\section{Discusión y conclusiones}

El análisis expuesto en este trabajo sobre el caso del Departamento de Salud de Torrevieja en España, en la Comunidad Valenciana, es útil para futuros PPP en zonas limítrofes con afluencias de extranjeros y evidencia lo siguiente.

La situación geográfica de los Departamentos de Salud influye sustancialmente en el perfil de pacientes que van a ser atendidos en los mismos. En el caso analizado en concreto, se trata de una población costera que en los meses estivales recibe más población nacional (de otras comunidades) y que durante todo el año tiene un elevado porcentaje de pacientes extranjeros. 
Dada la forma de gestión del Departamento (concesional en este caso), este fenómeno puede acarrear consecuencias económicas para la empresa gestora de la concesión y también para la propia Generalitat. En concreto, la concesionaria recibe la cápita fija de su población asignada, pero además recibe compensación por todas aquellas asistencias a pacientes que no son cápita pero que sí son atendidos en el hospital.

El perfil de la población no cápita del Departamento de Salud de Torrevieja se caracteriza por un gran número de pacientes residentes de países extranjeros. Estos pacientes viven durante todo el año en las zonas que se asignan al Departamento de Salud estudiado, sin apenas incrementarse durante la época estival.

En los meses de verano, este perfil de Departamento de Salud debe asumir una mayor carga asistencial a causa de los desplazamientos de la población nacional, así como extranjera. En estos periodos, el perfil de los pacientes atendidos es de turistas tanto nacionales como extranjeros.

\section{Referencias}

1. Ortún Rubio V. Reforma de la sanidad. In: Las estructuras del Bienestar Propuestas de reforma y nuevos horizontes. Madrid: Civitas; 2002. p.159-207.

2. Soler MLM, Vasallo JM. Sistema Nacional de Salud Español. Características y Análisis. Enfermería Glob. 2007;6(1): 1-14. Available from: http://revistas.um.es/eglobal/article/view/256

3. Caballer-Tarazona M, Clemente-Collado A, Vivas-Consuelo D. A cost and performance comparison of Public Private Partnership and public hospitals in Spain. Health Econ Rev. 2016;6(1): 1-7. http://dx .doi.org/10.1186/s13561-016-0095-5

4. López-Casasnovas G, del Llano-Señarís JE. Colaboración público-privada en sanidad: el modelo Alzira. Fundación Gaspar Casal (ed.) Fundación Gaspar Casal; 2018. 249 p.

5. Sánchez Bayle M. La privatización de la asistencia sanitaria en España; 2014. http://www.fundacionalternativas.org/public/storage/laboratorio_documentos_archivos/24bb 33c2b5a6aca0f1ea50a199da5de7.pdf

6. Rodríguez-Vigil Rubio JL. La descentralización del sistema sanitario español; 2012. 34 p. Madrid: Fundación Manuel Giméndez Abad.

7. Rey J. Aspectos generales y análisis del caso español y análisis del caso español; 1998. Granada: Escuela Andaluza de Salud Pública.

8. Ministerio de sanidad servicios sociales e igualdad. Sistema Nacional de Salud SNS. Minist Sanid Serv Soc e Igual; 2012. 54 p.

9. Perpiñán JMA. El sistema sanitario público en España y sus comunidades autónomas: sostenibilidad y reformas. Fundación BBVA; 2013. 406 p. 
10. Comunidad Valenciana. Ley 10/2014, de 29 de diciembre, de Salud de la Comunitat Valenciana. 2014, p. 1-85.

11. Acerete B, Stafford A, Stapleton P. Spanish healthcare public private partnerships: The "Alzira model." Crit perspect account. 2011;22(6): 533-549. http://dx.doi.org/10.1016/j.cpa.2011.06.004

12. Alonso JM, Clifton J, Díaz-Fuentes D. The impact of New Public Management on efficiency: An analysis of Madrid's hospitals. Health Policy (New York). 2015;119(3): 333-340. https://doi.org/10. 1016/j.healthpol.2014.12.001

13. Lopez-Casasnovas G, Costa-Font J, Planas I. Diversity and regional inequalities in the Spanish "system of health care services." Health Econ. 2005;14(suppl. 1): 221-235. https://doi.org/10.1002/hec.1038.

14. Wang H. et al. Public-private partnership in public administration discipline: A literature review. Public Manag Rev. 2017;20(2): 293-316. https://doi.org/10.1080/14719037.2017.1313445.

15. Li B, et al. Critical success factors for PPP/PFI projects in the UK construction industry. Constr Manag Econom. 2005;23(5): 459-471. https://doi.org/10.1080/01446190500041537.

16. Abuzaineh, N., Brashers, E., Foong, S., Feachem, R., Da Rita, P. PPPs in healthcare: Models, lessons and trends for the future. Healthcare public-private partnership series, No. 4. San Francisco: The Global Health Group, Institute for Global Health Sciences, University of California, San Francisco and PwC. Produced in the United States of America. 2018;(4), p. 53. https://globalhealthsciences.u csf.edu/public-private-partnerships-0.

17. Blasco Castany R. Las Nuevas Formas de Gestión santiaria - Modelo Alriza; 2007. http://laribera.ribe rasalud.com/wp-content/uploads/2016/11/Libro-Modelo-Alzira.pdf

18. Conselleria de Sanitat. Contrato de Gestión de Servicios Públicos por Concesión. Valencia; 2003.

19. Conselleria de Sanitat. IV Plan de Salud 2016-2020 Comunitat Valenciana; 2016. 238 p.

20. Comendeiro-Maaløe M, Ridao-López M, Gorgemans S, Bernal-Delgado E. Public-private partnerships in the Spanish National Health System: The reversion of the Alzira model. Health Policy (New York). 2019;13: 10-13. https://doi.org/10.1016/j.healthpol.2019.01.012

21. Diputación de Alicante. Datos del padrón municipal. Accesible en http://documentacion.diputacionali cante.es/4hogares.asp?codigo $=03133$

Notas

* $\quad$ Artículo de investigación

167.883 residentes británicos censados a finales de 2018 según INE, de los 248.824 de todo el país. 